\title{
Single-Center Experience With Liver Transplant Using Donors With Very High Transaminase Levels
}

\author{
Paulo N. Martins, ${ }^{1}$ Amanda Rawson, ${ }^{1}$ Babak Movahedi, ${ }^{1}$ Isabel M. A. Brüggenwirth, ${ }^{1,2}$ \\ Natasha H. Dolgin, ${ }^{1}$ Ann-Britt Martins, ${ }^{3}$ Paria Mahboub, ${ }^{1}$ Adel Bozorgzadeh ${ }^{1}$
}

\begin{abstract}
Objectives: Elevation of transaminases has been used as a marker of hepatic ischemic injury and as a crucial parameter for liver graft assessment. However, analysis of serum transaminases has limitations regarding the quantitative assessment of liver necrosis and is not a reliable predictor of outcomes.

Materials and Methods: We retrospectively reviewed the medical records of all liver transplants $(\mathrm{N}=238)$ performed at the UMass Memorial Medical Center from 2009 to 2013.
\end{abstract}

Results: Fourteen liver grafts showed high peak aminotransferases alanine aminotransferase (ALT) and aspartate aminotransferase (AST) at $>1000 \mathrm{U} / \mathrm{L}$. This high aminotransferase group was compared with 224 donors with low transaminase levels (ALT/AST $<1000 \mathrm{U} / \mathrm{L}$ ). The high transaminase donors had a median peak AST level of 3216 U/L (range, 1823-13030 $\mathrm{U} / \mathrm{L}$ ) and ALT level of $2677 \mathrm{U} / \mathrm{L}$ (range, 812-7080 U/L). The high transaminase donors showed higher levels of lactate dehydrogenase, international normalized ratio, total bilirubin, and gamma-glutamyltransferase compared with low transaminase donors; however, only lactate dehydrogenase results reached statistical significance. None of the grafts from the high transaminase donors showed primary nonfunction. Three-year graft and patient survival rates were similar in both groups $(75 \%$ vs $80 \%[P=.48]$ and $72 \%$ vs $82 \%$ $[P=.33]$, respectively). In an analysis of the discard rate of livers over a 10-year period in the United States using the Scientific Registry of Transplant Recipients

From the ${ }^{1}$ Department of Surgery, Division of Organ Transplantation, UMass Memorial Medical Center, University Campus, Worcester, Massachusetts, USA; the 2Department of Surgery, Division of Organ Transplantation, Groningen, The Netherlands; and the ${ }^{3}$ Department of Pediatrics, UMass Memorial Medical Center, University Campus, Worcester, Massachusetts, USA

Acknowledgements: This study was supported by the University of Massachusetts Institutional grant and AASLD Career Development Grant. The authors declare no conflicts of interest.

Corresponding author: Adel Bozorgzadeh, Department of Surgery, Transplant Division, University of Massachusetts, 55 Lake Ave North, Worcester, MA 01655, USA

Phone: +1 5083347408 E-mail: adel.bozorgzadeh@umassmemorial.org

Experimental and Clinical Transplantation (2018) database, the discard rate of livers with high aminotransferase levels was $69.14 \%$ compared with $22.23 \%$ for livers with low transaminase levels.

Conclusions: Liver grafts from donors with high transaminase levels can lead to clinical results that are similar to liver grafts from donors who had lower peak transaminase levels.

Key words: Alanine aminotransferase, Aspartate aminotransferase, Hepatic ischemic injury, Outcomes, Scientific Registry of Transplant Recipients

\section{Introduction}

The shortage of suitable donor livers is the most pressing problem facing liver transplantation today. Currently, in the United States, 16204 people are waiting for a liver transplant, and mortality on the wait list is $14 \% .^{1}$ This scenario has forced the extrapolation of transplantability criteria of liver grafts. Despite the fact that many livers are not procured, about $3 \%$ of procured livers are not transplanted because of concerns with organ quality or other associated risks. ${ }^{2}$ One frequent reason why many liver grafts are being discarded is the high level of transaminases in the donor. Serum aminotransferases, specifically, aspartate aminotransferase (AST) and alanine aminotransferase (ALT), are generally used as indicators of hepatocellular damage and liver cell necrosis. However, there are no clear upper limits of serum transaminases to contraindicate a transplant.

Hypoxic hepatitis, also known as ischemic hepatitis or shock liver, is the most frequent cause of notably raised aminotransferases in the hospital and intensive care unit. This acute liver injury is mainly caused by insufficient oxygen uptake by hepatocytes. Several mechanisms can lead to hypoxic hepatitis, including hepatic ischemia as a consequence of insufficient hepatic perfusion, insufficient oxygen 
extraction by the liver, systemic arterial hypoxemia, and passive venous congestion. ${ }^{3}$ An imbalance in metabolic supply and demand within the ischemic organ results in profound tissue hypoxia and microvascular dysfunction. Subsequent reperfusion during transplant procedures further enhances the activation of innate and adaptive immune responses and cell death programs. ${ }^{4,5}$

The goal of our study was to compare the clinical outcomes of liver transplants in our center from donors who had peak AST or ALT beyond $1000 \mathrm{U} / \mathrm{L}$ with donors with lower transaminase levels.

\section{Materials and Methods}

\section{Patients and study design}

This retrospective analyses and presentation of data from our transplant database were approved by the University of Massachusetts Medical School Institutional Review Board. This investigation involved 238 patients who underwent orthotopic liver transplant at UMass Memorial Medical Center from January 1, 2009 through June 9, 2013. The study population included patients over the age of 18 years at the time of first transplant who received whole liver allografts from brain-dead donors. Patients included in the study were divided into 2 groups according to the status of their donated allograft: (1) livers from donors with peak AST or ALT $>1000 \mathrm{U} / \mathrm{L}(\mathrm{n}=14)$ and $(2)$ livers from donors with peak AST or ALT $<1000 \mathrm{U} / \mathrm{L}(\mathrm{n}=224)$. This transaminase cut-off was chosen empirically to reflect a major increase of these values ( $>20$-times baseline). The median follow-up after transplant was 1766 days (mean [standard deviation] of 1701 [508.2] days).

Demographic and clinical characteristics of study subjects were obtained through a retrospective review of our center's electronic medical records. Information related to organ donors was collected from the United Network for Organ Sharing (UNOS) donor records using their DonorNet application. Model for End-Stage Liver Disease (MELD) scores utilized in this investigation were gathered from UNet (also from UNOS) and reflected the recipient's MELD score as listed with UNOS at the time of transplant. We could detect the exact position of the recipient in the liver transplant wait list by recording the match number. Donor liver biopsies were obtained at the time of procurement as frozen-section biopsies. Combined length of stay was calculated as the sum of the intensive care unit length of stay and hospital length of stay posttransplant. The donor risk index was calculated according to the formula specified by Feng and associates. ${ }^{6}$ Local organs were transplanted according to the MELD allocation policy. Imported organs, defined as an organ that was declined by all transplant centers from at least 1 UNOS region, were allocated according to our center's preference. Asystole was determined at the time the donor had no measurable pulse or was estimated when the patient was found unconscious prior to resuscitation.

All transplant recipients were listed according to standard protocols of our center and UNOS. All operations were performed using standard surgical techniques by the same surgical team. All livers were preserved in University of Wisconsin solution and implanted using piggyback technique without portocaval shunt. Immunosuppression consisted of a methylprednisolone bolus during the anhepatic phase followed by prednisone taper, mycophenolate mofetil, and tacrolimus.

Variables investigated included patient and graft survival, surgical complications (eg, hepatic artery thrombosis, reexploration rate, portal vein thrombosis, and biliary complications), primary nonfunction, early allograft dysfunction, postreperfusion syndrome, and posttransplant recipient liver function tests. Early allograft dysfunction was defined by the presence of one or more of the following: (1) bilirubin $\geq 10 \mathrm{mg} / \mathrm{dL}$ on day 7 , (2) international normalized ratio (INR) $\geq 1.6$ on day 7, and (3) AST or ALT > $2000 \mathrm{IU} / \mathrm{L}$ within the first 7 days. ${ }^{7}$ Postreperfusion syndrome was defined as a decrease in the mean arterial pressure of more than $30 \%$ of the value observed in the anhepatic stage, for more than 1 minute during the first 5 minutes after reperfusion of the graft. ${ }^{8}$ Primary nonfunction was defined as death or retransplant within 1 week after transplant associated with liver failure in the absence of vascular complications. ${ }^{9}$

In addition to our single-center study, we used the Scientific Registry of Transplant Patients (SRTR) database to analyze the discard rate of livers over a 10 -year period in the United States, taking into account donor high versus low transaminases levels (see Figure 5). In total, $98.22 \%$ of transplanted livers had transaminase levels $<1000 \mathrm{U} / \mathrm{L}$ compared with $1.78 \%$ of transplanted livers with transaminase levels $>1000 \mathrm{U} / \mathrm{L}$. 


\section{Statistical analyses}

Data collection and statistical analyses were performed using IBM SPSS Statistics (version 20.0; SPSS Inc., Chicago, IL, USA) software. Data are presented as mean and standard deviation for continuous variables unless all data were tested for normality using the method of Kolmogorov-Smirnov. Categorical variables were compared using chi-square tests, and continuous variables were analyzed using $t$ tests and analysis of variance where appropriate. Kaplan-Meier survival analysis was used to evaluate patient and graft survival, with the log-rank test used to assess differences between survival curves. Statistical significance was defined as $P<.05$.

\section{Results}

\section{Donor characteristics}

All 238 donors included in this analysis were brain dead. From this cohort, 14 donors had a peak AST or ALT of $>1000 \mathrm{U} / \mathrm{L}$ (Table 1). Before organ recovery, the median peak in this group was total bilirubin of $1.7 \mathrm{mg} / \mathrm{dL}$ (normal range from 0.3-1.9 U/L), gammaglutamyltransferase of $58 \mathrm{U} / \mathrm{L}$ (normal range from 0-45 U/L), lactate dehydrogenase of $1769 \mathrm{U} / \mathrm{L}$ (normal range from 140-280 U / L), INR of 2.5 (normal range from 0.8-1.2), AST of $3216 \mathrm{U} / \mathrm{L}$ (normal range from 10-40 U/L), and ALT of $2677 \mathrm{U} / \mathrm{L}$ (normal range from $7-56 \mathrm{U} / \mathrm{L})$. The median down time (asystole) was 30 minutes (range, 0 to 99 min). Eleven donor grafts $(78.5 \%)$ from the high transaminase group were imported from other regions after being declined by other centers. Most of these livers went to patients who were low on the list. The median donor match sequence for all 14 patients who received livers from high transaminase donors was
361 (range, 2-3506). Donors from the high transaminase group were younger than donors from the low transaminase group (median age of 40 vs 55 years; $P=.005)$. The donor risk index in high transaminase donors was lower than the risk index in low transaminase donors, but this did not reach statistical significance (1.89 vs $2.12 ; P=.1)$. In the high transaminase group, $83.3 \%$ died of anoxia, compared with $22.8 \%$ of low transaminase donors $(P=.001)$. We also found that high transaminase donors had higher levels of lactate dehydrogenase, INR, total bilirubin, and gamma-glutamyltransferase, but only lactate dehydrogenase levels reached statistical significance (1769 vs $325 \mathrm{U} / \mathrm{L} ; P<.001$ ).

Levels of AST and ALT usually peaked 24 to 48 hours before the time of organ recovery, when levels were on average less than $50 \%$ of peak levels. It is worth mentioning that, in this series, most donors did not have a significantly associated increase in INR or total bilirubin (Figure 1).

In all donors except for 2, a frozen-section liver biopsy was performed at the time of procurement. There were no signs of major steatosis, necrosis, or inflammation in these grafts. Only 1 graft showed $15 \%$ to $20 \%$ necrosis. Frozen-section biopsy has been shown to reliably assess inflammation and necrosis, correlating with results shown with permanentsection microscopy (Table 2). ${ }^{10}$ Donors were numbered from 1 to 14 in Table 2, and each donor donated a liver graft to the respective recipient numbered from 1 to 14 in Table 3.

\section{Recipient characteristics}

Recipient characteristics are summarized in Table 3. Average patient age, average MELD scores at listing, body mass index, sex, and ethnicity were similar in

\begin{tabular}{|c|c|c|c|c|c|c|c|c|c|}
\hline & COD & $\begin{array}{c}\text { Down } \\
\text { Time, min }\end{array}$ & $\begin{array}{c}\mathrm{AST}, \mathrm{U} / \mathrm{L} \\
\text { (first/peak/last) }\end{array}$ & $\begin{array}{l}\text { ALT, U/L (first, } \\
\text { peak, last) }\end{array}$ & $\begin{array}{c}\text { Tbili, mg/dL } \\
\text { (first, peak, last) }\end{array}$ & $\begin{array}{l}\text { INR (first, } \\
\text { peak, last) }\end{array}$ & $\begin{array}{c}\mathrm{pH} \text { (first, } \\
\text { lowest, last) }\end{array}$ & $\begin{array}{c}\text { Peak } \\
\text { Sodium, } \mathrm{mEq} / \mathrm{L}\end{array}$ & $\begin{array}{l}\text { CIT, } \\
\text { min }\end{array}$ \\
\hline 1 & Anoxia & 47 & $3030 / 13030 / 46$ & 4086/7080/150 & $0.2 / 1.1 / 0.2$ & 3.0/7.4/1.1 & $6.7 / 6.7 / 7.2$ & & 547 \\
\hline 2 & Anoxia & 43 & $3794 / 8464 / 7434$ & $1463 / 3958 / 3958$ & $0.4 / 1.7 / 1.7$ & 3.8/3.8/3.0 & 7.2/7.0/7.4 & 152 & 577 \\
\hline 3 & Anoxia & 19 & $44 / 7585 / 115$ & $27 / 2911 / 78$ & $0.6 / 3.9 / 3.7$ & $1.9 / 3.4 / 2.0$ & $7.4 / 7.1 / 7.4$ & 173 & 648 \\
\hline 4 & Anoxia & 99 & $556 / 5833 / 2887$ & $346 / 3395 / 2096$ & $0.3 / 2.0 / 2.0$ & $1.1 / 3.7 / 3.7$ & 7.2/7.1/7.2 & 135 & 508 \\
\hline 5 & Anoxia & none & $18 / 4912 / 124$ & $12 / 2561 / 387$ & $1.2 / 2.2 / 2.2$ & $1.0 / 1.9 / 1.2$ & 7.3/7.3/7.5 & 136 & 511 \\
\hline 6 & Anoxia & 30 & $1123 / 3295 / 1112$ & $292 / 754 / 469$ & $0.2 / 1.3 / 1.2$ & $1.2 / 2.9 / 2.8$ & 7.0/6.9/7.4 & 141 & 552 \\
\hline 7 & Anoxia & 46 & $76 / 3138 / 1710$ & $94 / 1062 / 939$ & $0.4 / 2.0 / 2.0$ & $2.0 / 2.9 / 2.8$ & $6.5 / 6.5 / 7.4$ & 148 & 540 \\
\hline 8 & Head trauma & None & $84 / 2794 / 1446$ & 44/2794/1714 & $0.4 / 1.1 / 0.7$ & $1.2 / 1.3 / 1.2$ & 7.3/7.1/7.4 & 152 & 600 \\
\hline 9 & Anoxia & 15 & $1204 / 2272 / 513$ & $1692 / 2331 / 688$ & $0.8 / 1.8 / 1.8$ & $1.1 / 2.5 / 1.5$ & $6.7 / 6.7 / 7.5$ & 155 & 540 \\
\hline 10 & Anoxia & 30 & $1500 / 1823 / 1823$ & $2003 / 2855 / 1828$ & $0.4 / 0.6 / 0.7$ & $2.5 / 2.5 / 1.7$ & 7.2/7.2/7.4 & 149 & 510 \\
\hline 11 & CVA & none & 1429/1429/372 & 296/812/337 & 1.7/1.9/1.1 & $1.2 / 1.1 / 1.2$ & 7.3/7.1/7.4 & 152 & 546 \\
\hline 12 & Anoxia & 40 & $1345 / 1345 / 371$ & $454 / 454 / 245$ & $0.7 / 0.7 / 0.4$ & $1.2 / 1.5 / 1.5$ & 7.1/7.1/7.2 & 141 & 468 \\
\hline 13 & Head trauma & none & $540 / 1239 / 1221$ & 243/794/794 & $0.3 / 0.7 / 0.6$ & $1.8 / 1.8 / 1.4$ & 7.2/7.1/7.4 & 145 & 585 \\
\hline 14 & Anoxia & 80 & 434/1096/138 & 206/234/109 & $0.3 / 0.5 / 0.5$ & 1.0/1.1/1.0 & 7.0/7.0/7.4 & 138 & 480 \\
\hline
\end{tabular}

Abbreviations: ALT, alanine aminotransferase; AST, aspartate aminotransferase; CIT, cold ischemia time; COD, cause of death; CVA, cerebrovascular accident; INR, international normalized ratio; Tbili, total bilirubin 
recipients of grafts from high versus low transaminase donors. The median MELD score of livers with elevated transaminase was 19.4. Indications for transplant and immunosuppression were comparable in both groups. As mentioned above, recipients were numbered from 1 to 14 in Table 3, and each recipient received a liver graft from the respective donor numbered from 1 to 14 in Table 2.

\section{Transplant outcomes}

There were no differences in surgical complications (eg, hepatic artery thrombosis, reexploration rate, portal vein thrombosis, biliary complications), intensive care unit and hospital stay (4 vs 4 days $[P=.87]$ and 12.5 vs 10.5 days $[P=.58]$ ), and use of blood products during the first 24 hours (including the transplant procedure) between groups. None of the livers from the high transaminase group had

\begin{tabular}{|c|c|}
\hline Donor & Biopsy Result \\
\hline 1 & $\begin{array}{l}<5 \% \text { macrosteatosis, } 0 \% \text { microsteatosis, no necrosis, no } \\
\text { inflammation }\end{array}$ \\
\hline 2 & $\begin{array}{l}2 \% \text { macrosteatosis, microsteatosis mild, necrosis }<1 \% \text {, } \\
\text { inflammation } 1 / 4\end{array}$ \\
\hline 3 & $\begin{array}{l}5 \% \text { macrosteatosis, } 10 \% \text { microsteatosis, bridging centrolobular } \\
\text { necrosis } 15 \% \text { to } 20 \%\end{array}$ \\
\hline 4 & no steatosis, mild inflammation, no necrosis \\
\hline 5 & $\begin{array}{l}<5 \% \text { macrosteatosis, } 15 \% \text { to } 20 \% \text { microsteatosis, no inflammation, } \\
\text { no necrosis, cholestasis }\end{array}$ \\
\hline 6 & no steatosis, no inflammation, no necrosis \\
\hline 7 & no steatosis, no inflammation, no necrosis, cholestasis \\
\hline 8 & no steatosis, no inflammation, no necrosis, cholestasis \\
\hline 9 & $\begin{array}{l}<5 \% \text { macrosteatosis, }<5 \% \text { microsteatosis, no necrosis, mild } \\
\text { inflammation, cholestasis }\end{array}$ \\
\hline 10 & $<5 \%$ macrosteatosis, no inflammation, no necrosis \\
\hline 11 & minimal steatosis, mild inflammation, no necrosis, cholestasis \\
\hline 12 & $\begin{array}{l}10 \% \text { to } 15 \% \text { macrosteatosis, } 10 \% \text { to } 15 \% \text { microsteatosis, no } \\
\text { necrosis, no inflammation }\end{array}$ \\
\hline 13 & no steatosis, mild inflammation, no necrosis, cholestasis \\
\hline 14 & no biopsy \\
\hline
\end{tabular}

Figure 1. Donor Liver Function Tests From Admission to Day of Procurement in 14 Donors With High Transaminase Levels

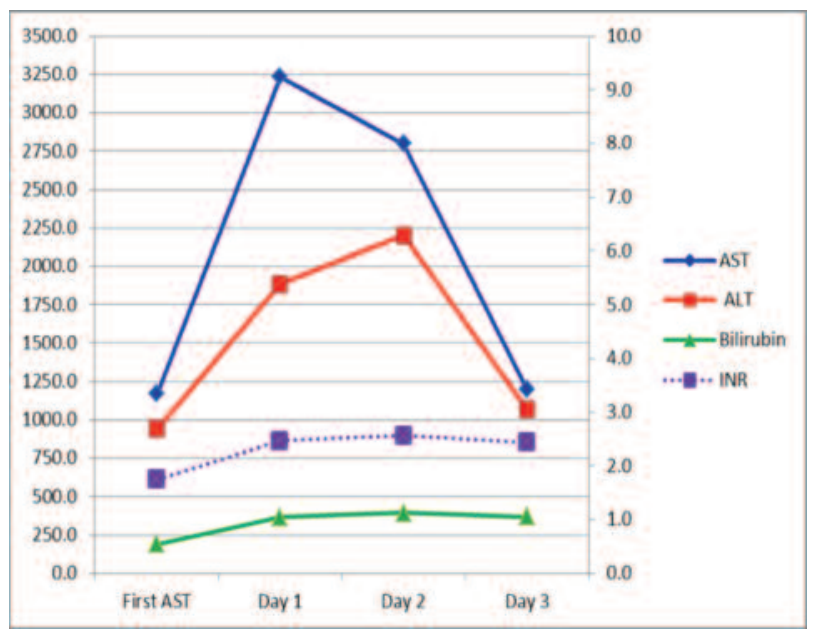

Aspartate aminotransferase (AST) and alanine aminotransferase (ALT) usually peaked 24 to 48 hours after the initial ischemic insult and decreased thereafter. Those donors did not show increase in international normalized ratio (INR) and total bilirubin before procurement.

primary nonfunction (no significant difference from the low transaminase group). The rate of early allograft dysfunction was $14 \%$, which is similar to control and data in the literature. ${ }^{11-13}$ Liver function tests of recipients during the first week showed that these livers recovered fast, with most of these parameters getting close to normal levels within the first week (Figure 2). The rate of postreperfusion syndrome was $20 \%$. None of these 14 patients presented with severe biliary complications. Of the 14 patients, $5(36 \%)$ underwent endoscopic retrograde cholangiopancreatograms, but only $3(21 \%)$ had anastomotic strictures that were treated successfully with stents.

Figure 3 shows Kaplan-Meyer patient survival curves for both groups of patients. The 1- and 3-year survival curves of the 14 recipients of high

\begin{tabular}{|c|c|c|c|c|c|c|c|c|c|c|}
\hline Sex & Ethnicity & MELD & Diagnosis & $\mathrm{HCC}$ & $\begin{array}{c}\text { Donor Match } \\
\text { Sequence }\end{array}$ & IPF & Follow-up, d & LOS, d & $\begin{array}{c}\text { Number of } \\
\text { of PRBC in OR }\end{array}$ & $\begin{array}{l}\text { Number } \\
\text { of ERCPs }\end{array}$ \\
\hline$M$ & W & 10 & $\mathrm{ETOH}$ & No & 2.708 & No & 1923 & 11 & 0 & 0 \\
\hline M & $\mathrm{H}$ & 17 & $\mathrm{ETOH}$ & No & 2.026 & No & 1787 & 10 & 5 & 0 \\
\hline M & $\mathrm{H}$ & 13 & $\mathrm{ETOH}$ & No & 2.374 & Yes & 1892 & 4 & 7 & 0 \\
\hline$M$ & W & 17 & $\mathrm{NASH}$ & No & 386 & No & 1784 & 34 & 6 & 4 \\
\hline M & W & 19 & $\mathrm{ETOH}$ & No & 335 & No & 1091 & 15 & 9 & 0 \\
\hline $\mathrm{F}$ & W & 12 & $\mathrm{HCV} / \mathrm{ETOH}$ & No & 2.483 & No & 1373 & 17 & 11 & 0 \\
\hline M & W & 25 & $\mathrm{HCV}$ & Yes & 170 & No & 1433 & 19 & 12 & 2 \\
\hline$M$ & W & 22 & $\mathrm{HCV}$ & Yes & 17 & No & 2419 & 7 & 4 & 0 \\
\hline$M$ & W & 16 & $\mathrm{HCV}$ & No & 33 & No & 1747 & 19 & 4 & 0 \\
\hline $\mathrm{F}$ & W & 31 & $\mathrm{HCV} / \mathrm{ETOH}$ & No & 17 & No & 2039 & 15 & 7 & 3 \\
\hline M & W & 15 & $\mathrm{NASH}$ & No & 3.506 & No & 1332 & 20 & 6 & 3 \\
\hline M & W & 23 & $\mathrm{ETOH}$ & No & 1.110 & No & 1172 & 9 & 8 & 0 \\
\hline $\mathrm{F}$ & W & 28 & $\mathrm{HCV}$ & No & 12 & No & 1028 & 15 & 3 & 0 \\
\hline M & W & 24 & $\mathrm{HCV} / \mathrm{ETOH}$ & Yes & 2 & Yes & 2799 & 21 & 4 & 2 \\
\hline
\end{tabular}

Abbreviations: ERCP, endoscopic retrograde cholangiopancreatogram; ETOH, alcoholic hepatitis; F, female; HCC, hepatocellular carcinoma; H, Hispanic; HCV, hepatitis C virus; IPF, initial poor function; LOS, length of stay; M, male; MELD, Model for End-Stage Liver Disease; NASH, nonalcoholic steatohepatitis; OR, operation room; PRBC, packed red blood cells; W, white 
Figure 2. Recipient Transaminases, Total Bilirubin, Alkaline Phosphatase, and International Normalized Ratio in the First 7 Days Posttransplant in the High Transaminase Group $(n=14)$
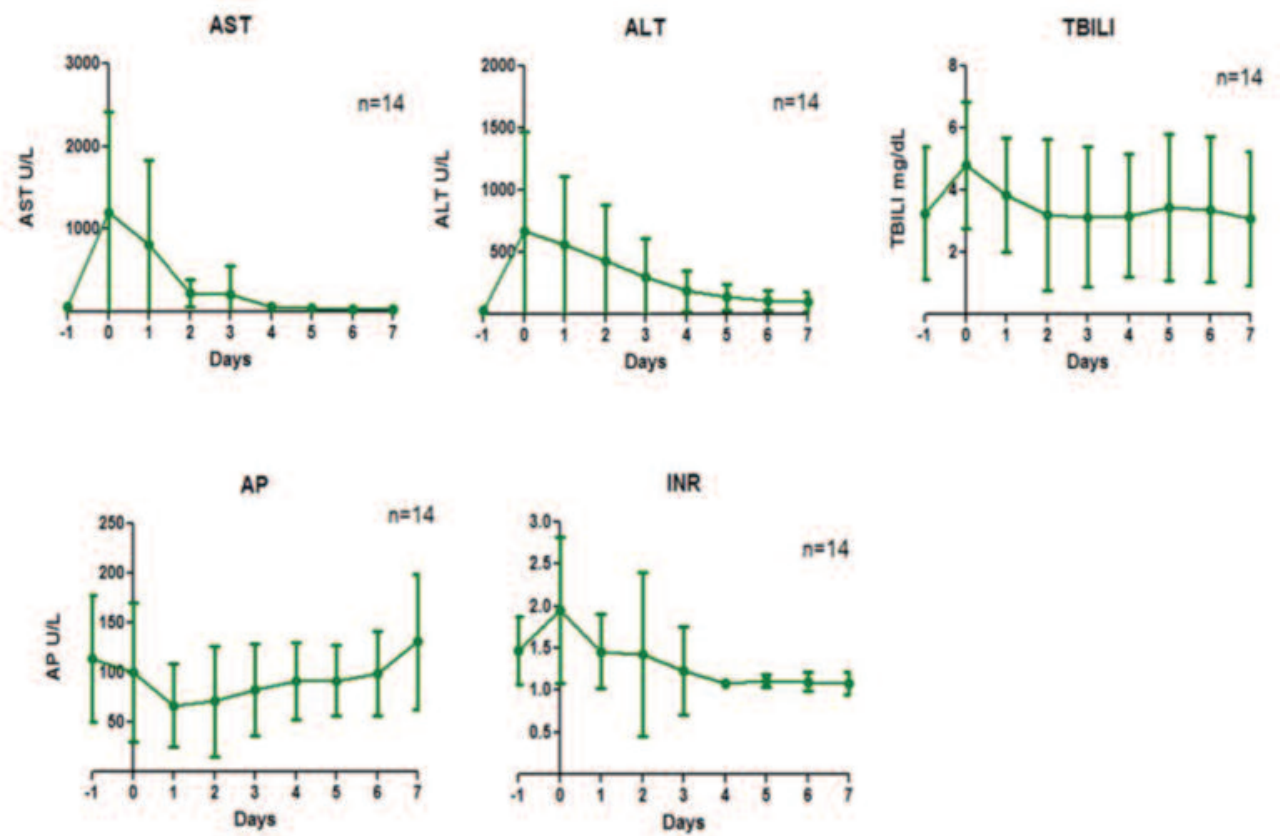

Abbreviations: ALT, alanine aminotransferase; AST, aspartate aminotransferase; AP, alkaline phosphatase; INR, international normalized ratio; TBILI, total bilirubin

Figure 3. Survival Curves of Patients Who Received Organs From Donors With High Transaminase Versus Lower Transaminase Levels

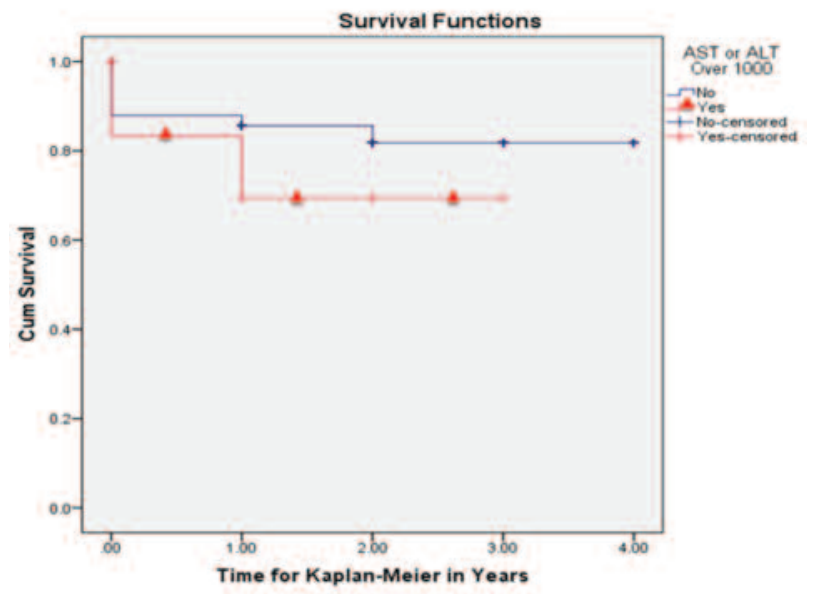

Abbreviations: ALT, alanine aminotransferase; AST, aspartate aminotransferase; AP, alkaline phosphatase; INR, international normalized ratio; TBILI, total bilirubin

transaminase donor livers were worse than those in the 224 recipients of low transaminase donor livers (85\% vs $88 \%[P=.332]$ and $71 \%$ vs $84 \%$ [ $P=.08]$, respectively), but this did not reach statistical significance. Graft survival was not significantly different between the high transaminase and low transaminase groups (1-year graft survival of $85 \%$ vs $87 \%[P=.483]$ and 3-year graft survival of $71 \%$ vs $83 \%[P=.12]$ ) (Figure 4$)$.
Figure 4. Survival Curves of Grafts From Donors With High Transaminase Versus Lower Transaminase Levels

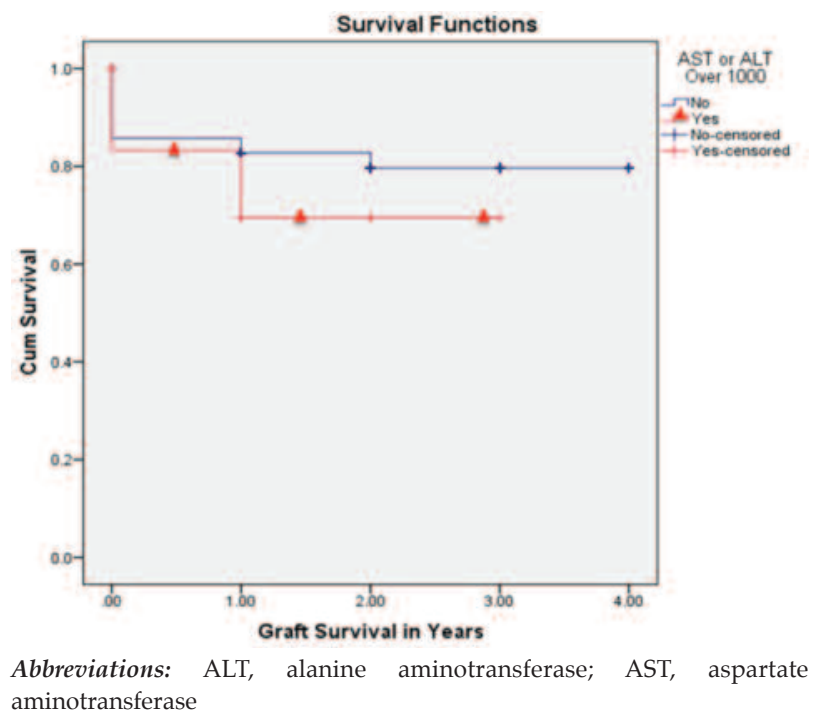

In the SRTR database analyses, we found that, in the United States, $1.78 \%$ of all liver grafts offered for transplant between 2002 and 2012 had either AST or ALT above $1000 \mathrm{U} / \mathrm{L}$. The discard rate for these livers was $69.14 \%$ compared with $22.23 \%$ for low transaminase donors $(<1000 \mathrm{U} / \mathrm{L})$. We noted a decreased discard rate of livers with high transaminase levels over time after implementation of the MELD allocation system in February 2002 (Figure 5). 
Figure 5. Discard Rate of Livers From Donors With High Transaminases Over Time in the US Scientific Registry of Transplant Recipients Between 2002 and 2012

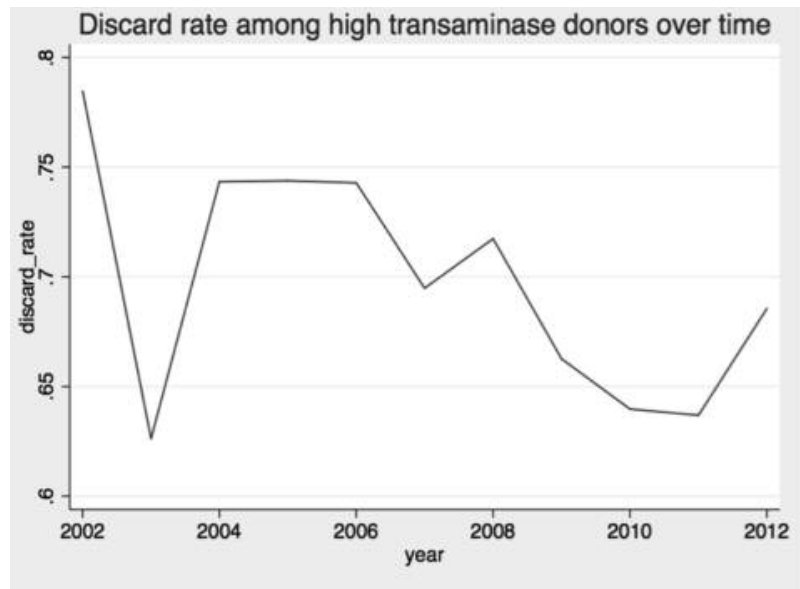

\section{Discussion}

Many usable liver grafts are being discarded unnecessarily. Tector and associates ${ }^{2}$ showed that $68 \%$ of recipients in their series (388 of 571) received expanded criteria donor grafts. Patient and graft survival rates were similar between recipients of standard criteria organs and expanded criteria donor grafts (1-year graft survival of $84 \%$ vs $80 \%$ and 2-year graft survival of $78 \%$ vs $77 \%$, respectively). In addition to good patient and graft outcomes, they showed an increase in the percentage of wait list removals for transplant $(P<.01)$, decrease in the percentage of removals for death or too sick to transplant $(P=.04)$, and increased rate of transplants per patient per year on the wait list compared with the national average. ${ }^{2}$

Throughout the body, AST and ALT levels are widely distributed. Although AST is found primarily in heart, liver, skeletal muscle, and kidney, ALT is found primarily in liver and kidney, with lesser amounts in heart and skeletal muscle. In addition, ALT is exclusively cytoplasmic, whereas AST is both mitochondrial and cytoplasmic in all cells. ${ }^{14}$ Traditionally, elevation of transaminases has been broadly used as a marker of hepatic ischemic injury and as a crucial parameter for liver graft assessment. However, serum transaminase analysis has limitations regarding quantitative assessment of liver necrosis. It is well established that levels of AST and ALT do not necessarily reflect the underlying necroinflammatory activity. ${ }^{15}$

Indeed, several factors other than liver disease (eg, muscle injury, heart infarct, hemolysis) can increase AST and ALT activities. ${ }^{16}$ These conditions are frequent in donors who have died secondary to trauma or a cardiac event. In addition, these levels may not reflect the number of necrotic cells. These limitations seem to result from instability of cellular enzymes. Clearance depends on the rate at which the enzymes are released from cells and the rate at which they are taken up by sinusoidal-lining cells. ${ }^{17}$ Therefore, the serum level observed in a single assay does not necessarily reflect the total amount released or the extent of ischemic damage. The half-life of AST in the circulation is $17 \pm 5$ hours, whereas that of ALT is $47 \pm 10$ hours. ${ }^{16}$ Therefore, it is necessary to consider the curve of transaminases and not only the peak when considering organs from donors with high transaminase levels. The typical pattern of transaminase elevation during the course of ischemic insult shows an initial dramatic rise of both transaminase and lactate dehydrogenase levels within 12 to 24 hours after the initiating event. ${ }^{18}$ Fuhrmann and associates showed that increasing AST levels for more than 24 hours is associated with worse liver injury. ${ }^{5}$ Usually, aminotransferase levels fall more than $50 \%$ within 3 days after stabilization and elimination of the underlying causing condition. Specifically, AST levels decline toward baseline levels earlier than ALT levels during this recovery period because of the shorter half-life of AST. ${ }^{16}$ It is also important to consider other tissue sources to explain the increase of transaminases, including trauma, hemolysis, and heart attack. Likewise, it is important to consider that other abnormal liver function tests associated with high transaminase levels can portend major liver insult (eg, high INR, high total bilirubin). In these cases, a liver biopsy before organ procurement or at the time of procurement can be of crucial importance in the decision-making process to accept or discard the graft.

Many organs from donors with increasing transaminases are discarded because increased AST / ALT results are usually considered surrogates of liver injury/necrosis. It has been shown that the presence of ischemic hepatitis in critically ill patients is associated with a 30-day mortality of more than $50 \% .5$ It is extremely common to find elevated transaminases during events that lead to brain-death donation. However, it is not clear to what extent the level of transaminases of the donor can predict clinical outcomes after transplant. A 2008 consensus conference addressing the use of expanded criteria 
donor grafts came to the conclusion that there is no clear upper limit above which the organ should not be transplanted and that liver procurement should not be excluded based on these enzymes alone. ${ }^{19}$

\section{Clinical evidence of the impact of transaminase level and outcomes}

Despite the fact that some single-center reports have shown correlations between absolute transaminase values and outcomes, $, 12,20$ it is generally accepted that these values are not good predictors of outcome. In a Cox regression analysis of 52 donor variables on liver graft survival of 5150 liver transplants, there was no negative effect when transaminases were above 200 $\mathrm{U} / \mathrm{L} .{ }^{21}$ In a Cox regression analysis by Feng and associates of SRTR data that included 20023 liver transplants, donor AST levels were not predictors of graft outcome; thus, donor AST level was not incorporated in the donor risk index. ${ }^{6}$ Likewise, the European donor risk index does not include donor AST as a predictor of liver graft outcomes. ${ }^{22}$ The levels of recipient transaminases posttransplant are also poor predictors of graft outcomes. Using a Cox regression analysis of 213 consecutive transplants, Rosen and associates showed that AST is predictive of graft survival only when it is markedly elevated (AST maximum $>5000 \mathrm{U} / \mathrm{L}$ ). ${ }^{23}$

However, in a predictor score system (SOFT score) that included transaminases in the risk calculation, Rana and associates found that AST > $140 \mathrm{IU} / \mathrm{L}$ and ALT > $100 \mathrm{IU} / \mathrm{L}$ were risk factors for worse outcome. ${ }^{24}$

There are 2 previous single-center studies that specifically investigated the impact of elevated transaminases as the primary variable. The first retrospective study was from Radunz and colleagues from Essen, Germany, who reported their experience with transplants of livers from 8 brain-dead donors who had markedly elevated levels of AST (median of $1400 \mathrm{U} / \mathrm{L}$; range 500-7538 U/L) and ALT (median of $1026 \mathrm{U} / \mathrm{L}$; range, 308-9179 U/L). In their series, liver enzymes had decreased in 6 of 8 donors, but there was no associated significant steatosis. None of these grafts had primary nonfunction, and all patients were alive at follow-up with the exception of 1 who died because of unrelated issues. Protocol biopsies at 3 to 6 months posttransplant showed portal fibrosis grade 1 in 3 patients and grade 2 in 1 patient. ${ }^{25}$

The second report was by Mangus and associates from the United States. In this retrospective study of
1348 consecutive deceased donor (including 64 donors after cardiac death) transplants, the recipients were categorized into 3 groups based on the elevation of serum ALT: normal/mild elevation (0-499 U / L), moderate elevation (500-999 U/L), and severe elevation $(\geq 1000 \mathrm{U} / \mathrm{L})$. In this cohort, there were 1259 patients included in the normal/mild group (93\%), 34 in the moderate group (3\%), and 55 in the severe group (4\%). Results from this analysis failed to demonstrate any differences in early graft function or graft and patient survival based on evaluation of peak donor ALT. Although $49 \%$ of the liver grafts with elevated donor ALT demonstrated evidence of hepatocyte necrosis on reperfusion biopsies, there was no correlation with peak of recipient serum transaminases after transplant. Subgroup analysis did not show an association between percent of total graft steatosis and higher donor ALT levels or increased risk of hepatocyte necrosis. The authors also found that ALT elevation was more associated with anoxia as a cause of death, and there was a direct association with the duration of donor asystole time. ${ }^{26}$

\section{Graft and recipient matching for livers with high transaminase levels}

We preferred to allocate livers with high transaminase levels to recipients with lower MELD scores and poor quality of life (despite the fact that in our series there was no statistically significant difference in MELD scores between the 2 groups). The rationale is that high MELD patients with major portal hypertension and associated comorbidities are less likely to tolerate a rough reperfusion than low MELD patients. ${ }^{27}$

Donors from the high transaminase group were significantly younger than donors in the low transaminase group. We are more hesitant to accept livers with high transaminases from older donors, since older age is a significant risk factor for poor outcomes. ${ }^{28}$ In addition, it is well-known that older age increases susceptibility of livers to ischemia. ${ }^{29-31}$ We also try to expedite allocation of donors with high transaminases to decrease the cold ischemia time and minimize ischemic damage. Despite the fact that most of these organs came from another region and needed first to be declined by all transplant centers of one region, our cold ischemia time was relatively short $(9.0 \mathrm{~h} \pm 46 \mathrm{~min}$, mean $\pm \mathrm{SD})$. These livers could be successfully allocated to recipients low on the list 
(median donor match sequence for all 14 patients who received livers from high transaminase donors was 361), going to a patient as low as the 3506th position on the wait list.

The main limitation of our study was the retrospective nature of the analysis and the fact that the number of donors with elevated transaminases (AST or ALT $>1000 \mathrm{U} / \mathrm{L})$ was small $(\mathrm{n}=14)$. In addition, nonrandomized studies have unavoidable biases related to organ allocation. The allocation system for imported organs (11 of 14) was not based on MELD score but based on "rescue allocation," since many were open offers and allocated to less sick patients (with lower MELD scores). In addition, we did not perform postreperfusion biopsies, and we have not tried to correlate transaminase levels with pretransplant biopsy results because of the small number of cases and the fact that only 1 liver showed necrosis. It is also well known that frozen-section biopsies have limitations regarding determination of degree of steatosis and fibrosis. Our analysis did not include the number of organ offers to our center from donors who had high AST or ALT and were not accepted for transplant. In addition, it is not possible to determine whether the presence of high transaminase levels was the main reason for declining a liver graft by other centers since the reasons for refusal in the UNOS organ allocation software application are generic and the true refusal reasons could have been a combination of unfavorable donor characteristics. We also did not analyze the history of alcohol abuse in this population. Another limitation was that many times it was impossible to determine exactly when asystole happened, and it was usually estimated when the patient was found unconscious before resuscitation.

Our series of livers from donors with high transaminase levels showed that these livers can be safely used and demonstrated results similar to those from 2 other centers. ${ }^{25,26}$ Although the number of patients in the study arm was small, our data demonstrated similar patient and graft survival between recipients of organs from donors with high transaminase and low transaminase levels. We showed here that it is feasible to use liver grafts from donors with markedly elevated AST/ALT levels with reasonable results. Our analyses of the SRTR database showed that the discard rate of these livers has been declining over time, possibly reflecting the increasing gap between demand and supply of organs and the increasing experience with standard criteria livers. Donors with high transaminase levels can be safely used to increase the organ donor pool. Judicious selection of these grafts, by eliminating additional risk factors (older age, significant steatosis, and necrosis), and expeditious allocation may contribute to favorable outcomes. The effects of adequate recipient matching require further characterization when using these organs.

\section{References}

1. United Network for Organ Sharing. Web site. https://www. unos.org/. Accessed May 4, 2016.

2. Tector AJ, Mangus RS, Chestovich P, et al. Use of extended criteria livers decreases wait time for liver transplantation without adversely impacting posttransplant survival. Ann Surg. 2006; 244(3):439-450

3. Birrer R, Takuda Y,Takara T. Hypoxic hepatopathy: pathophysiology and prognosis. Intern Med. 2007;46(14):1063-1070.

4. Henrion J, Schapira M, Heller FR. Ischemic hepatitis: the need for precise criteria. J Clin Gastroenterol. 1996;23(4):305.

5. Fuhrmann V, Kneidinger N, Herkner $H$, et al. Hypoxic hepatitis: underlying conditions and risk factors for mortality in critically ill patients. Intensive Care Med. 2009;35(8):1397-1405.

6. Feng S, Goodrich NP, Bragg-Gresham JL, et al. Characteristics associated with liver graft failure: the concept of a donor risk index. Am J Transplant. 2006;6(4):783-790.

7. Olthoff KM, Kulik L, Samstein B, et al. Validation of a current definition of early allograft dysfunction in liver transplant recipients and analysis of risk factors. Liver Transpl. 2010;16(8):943-949.

8. Paugam-Burtz C, Kavafyan J, Merckx P, et al. Postreperfusion syndrome during liver transplantation for cirrhosis: outcome and predictors. Liver Transpl. 2009;15(5):522-529.

9. Clavien PA, Camargo CA, Croxford R, Langer B, Levy GA, Greig PD. Definition and classification of negative outcomes in solid organ transplantation. Application in liver transplantation. Ann Surg. 1994;220(2):109-120

10. Lo IJ, Lefkowitch JH, Feirt N, et al. Utility of liver allograft biopsy obtained at procurement. Liver Transpl. 2008;14(5):639-646.

11. Nanashima A, Pillay P, Verran DJ, et al. Analysis of initial poor graft function after orthotopic liver transplantation: experience of an australian single liver transplantation center. Transplant Proc. 2002;34(4):1231-1235.

12. Maring JK, Klompmaker IJ, Zwaveling JH, Kranenburg $\mathrm{K}$, Ten Vergert EM, Slooff MJ. Poor initial graft function after orthotopic liver transplantation: can it be predicted and does it affect outcome? An analysis of 125 adult primary transplantations. Clin Transplant. 1997;11(5 Pt 1):373-379.

13. Strasberg SM, Howard TK, Molmenti EP, Hertl M. Selecting the donor liver: risk factors for poor function after orthotopic liver transplantation. Hepatology. 1994;20(4 Pt 1):829-838.

14. Wroblewski F. The clinical significance of alterations in transaminase activities of serum and other body fluids. Adv Clin Chem 1958;1(2):313-351.

15. Al-Chalabi T, Underhill JA, Portmann BC, McFarlane IG, Heneghan MA. Effects of serum aspartate aminotransferase levels in patients with autoimmune hepatitis influence disease course and outcome. Clin Gastroenterol Hepatol. 2008;6(12):1389-1395.

16. Dufour DR, Lott JA, Nolte FS, Gretch DR, Koff RS, Seeff LB. Diagnosis and monitoring of hepatic injury. II. Recommendations for use of laboratory tests in screening, diagnosis, and monitoring. Clin Chem. 2000;46(12):2050-2068.

17. Idéo G, De Franchis R, Bellobuono A, Tornaghi G. Aspartate aminotransferase isoenzymes in human serum in various liver diseases. Enzyme. 1971;12(5):529-536. 
18. Ebert EC. Hypoxic liver injury. Mayo Clin Proc. 2006;81(9):12321236.

19. Durand F, Renz JF, Alkofer B, et al. Report of the Paris consensus meeting on expanded criteria donors in liver transplantation. Liver Transpl. 2008;14(12):1694-1707.

20. Heise M, Settmacher U, Pfitzmann $R$, et al. A survival-based scoring-system for initial graft function following orthotopic liver transplantation. Transpl Int. 2003;16(11):794-800.

21. Cuende N, Miranda B, Cañón JF, Garrido G, Matesanz R. Donor characteristics associated with liver graft survival. Transplantation. 2005;79(10):1445-1452.

22. Braat $A E$, Blok JJ, Putter $H$, et al. The Eurotransplant donor risk index in liver transplantation: ET-DRI. Am J Transplant. 2012;12(10): 2789-2796.

23. Rosen HR, Martin P, Goss J, et al. Significance of early aminotransferase elevation after liver transplantation. Transplantation. 1998;65(1):68-72.

24. Rana A, Hardy MA, Halazun KJ, et al. Survival outcomes following liver transplantation (SOFT) score: a novel method to predict patient survival following liver transplantation. Am J Transplant. 2008:8(12):2537-2546.

25. Radunz S, Paul A, Nowak K, Treckmann JW, Saner FH, Mathé Z. Liver transplantation using donor organs with markedly elevated liver enzymes: how far can we go? Liver Int. 2011;31(7):1021-1027.
26. Mangus RS, Fridell JA, Kubal CA, Davis JP, Tector AJ. Elevated alanine aminotransferase (ALT) in the deceased donor: impact on early post-transplant liver allograft function. Liver Int. 2015;35(2): 524-531.

27. Johnson SR, Alexopoulos S, Curry M, Hanto DW. Primary nonfunction (PNF) in the MELD Era: An SRTR database analysis. Am J Transplant. 2007;7(4):1003-1009.

28. Busquets J, Xiol X, Fiqueras J, et al. The impact of donor age on liver transplantation: influence of donor age on early liver function and on subsequent patient and graft survival. Transplantation. 2001;71(12):1765-1771.

29. Reese PP, Sonawane SB, Thomasson A, Yeh H, Markmann JF. Donor age and cold ischemia interact to produce inferior 90-day liver allograft survival. Transplantation. 2008:85(12):1737-1744.

30. Deschênes M, Forbes $C$, Tchervenkov J, et al. Use of older donor livers is associated with more extensive ischemic damage on intraoperative biopsies during liver transplantation. Liver Transpl Surg. 1999;5(5):357-361.

31. Washburn WK, Johnson LB, Lewis WD, Jenkins RL. Graft function and outcome of older ( $>$ or $=60$ years) donor livers Transplantation. 1996;61(7):1062-1066. 